\title{
Successful rechallenge with cetuximab after an infusion related reaction to panitumumab in a patient with locally advanced rectal cancer
}

\author{
Hideyuki Yokokawa ${ }^{1,2}$ (D) Teppei Kono $^{1,2} \cdot$ Hiroaki Shidei $^{1} \cdot$ Kunihiro Oyama $^{1} \cdot$ Yoshitomo Ito $^{1} \cdot$ Rie Imaizumi $^{1}$. \\ Yutaka Miyano $^{1} \cdot$ Shunichi Shiozawa ${ }^{2} \cdot$ Kazuhiko Yoshimatsu $^{1,2}$
}

Received: 26 August 2020 / Accepted: 12 October 2020 / Published online: 30 October 2020

(C) The Author(s) 2020

\begin{abstract}
Incidence of infusion related reaction (IR) is more common with cetuximab (Cmab) than with panitumumab (Pmab). Although little is known about rechallenge IR with monoclonal antibodies, we experienced a successful rechallenge to Cmab after IR to Pmab. A 67-year-old female patient was scheduled for chemotherapy with mFOLFOX6 plus Pmab against unresectable advanced rectal cancer in the hope of tumor shrinkage. On the first administration of Pmab, she complained of dyspnea with shortness of breath and wheezing, even after premedication with steroids and antihistamines. Her reaction was judged as Grade 2 IR to Pmab. For the next course, we tried Cmab. No IRs were observed. Since then, she has undergone seven further courses of treatment, followed by surgical resection. The patient benefited from administration of Cmab after experiencing IR to Pmab, suggesting this treatment to be an option for patients of this type who experience IR to Pmab.
\end{abstract}

Keywords Infusion related reaction $\cdot$ Panitumumab $\cdot$ Cetuximab

\section{Introduction}

Cetuximab (Cmab) is a chimeric antibody that binds to epidermal growth factor receptor (EGFR) with murine fraction variable regions [1], whereas panitumumab (Pmab) is the first complete human monoclonal antibody similar to $\mathrm{Cmab}$ [2]. They both inhibit the proliferation and differentiation of EGFR-expressing normal and neoplastic cells and cause apoptosis. The incidence of documented infusion related reaction (IR) is more common with Cmab (all grades $(\mathrm{G})$ 15-21\%, G 3/4 2-5\%) than with Pmab (all G 4\%, G 3/4 1\%) [3-9]. Although anecdotal reports suggest successful

Hideyuki Yokokawa

yokokawa.hideyuki@twmu.ac.jp

Kazuhiko Yoshimatsu

kyoshsu@twmu.ac.jp

1 Department of Surgery, Saitama-ken Saiseikai Kurihashi Hospital, 714-6 Koemon, Kuki, Saitama 349-1105, Japan

2 Department of Surgery, Tokyo Women's Medical University, Medical Center East, 2-1-10 Nishiogu, Arakawa, Tokyo 116-8567, Japan rechallenge with Pmab following IR to Cmab [10], there are few known cases that show the opposite pattern [11].

We herein present a case of locally advanced rectal cancer that was successfully rechallenged with $\mathrm{Cmab}$ after IR to Pmab, followed by surgical resection.

\section{Case report}

A 67-year-old female patient was referred to our hospital with tenesmus and frequent bowel movements. Physical and hematochemical examinations revealed loss of body weight, malnutrition and inflammatory changes. Computed tomography (CT) scan revealed a rectal cancer that had developed to the external wall of the rectum and invaded the surrounding tissue (Fig. 1). On admission, induction of chemotherapy was conducted after stoma creation due to the unresectable nature of the tumor. Due to its identification as a wild-type tumor incorporating rat sarcoma viral oncogene homolog (Ras)/v-raf murine sarcoma viral oncogene homolog B (BRAF) genes, a combination of oxaliplatin, 5-fluorouracil and leucovorin (mFOLFOX6) plus Pmab was selected in the hope of causing rapid tumor shrinkage. As pretreatment before initiation of chemotherapy, $1.65 \mathrm{mg}$ of 
Fig. 1 Abdomino-pelvic CT scan on admission. The tumor originating from the rectum had grown into the extra-rectal wall (white arrow: a) and was suspected of having invaded the gluteus maximus (white arrow: b)

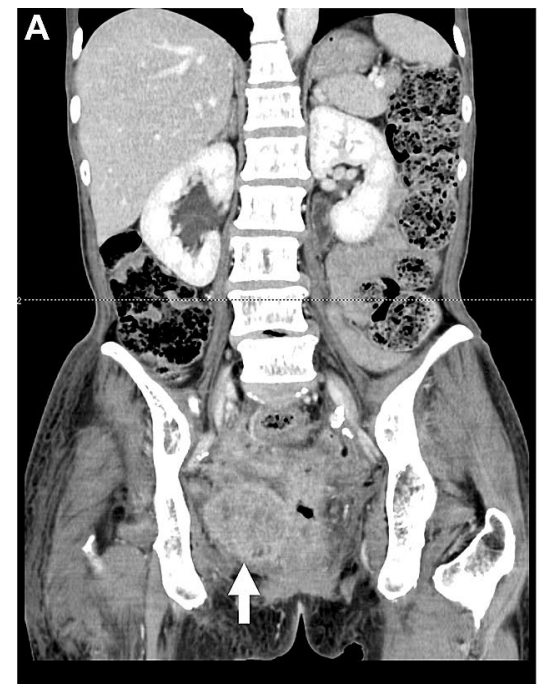

dexamethasone sodium phosphate and $5 \mathrm{mg}$ of chlorpheniramine maleate were injected to prevent adverse events including allergy. Then $260 \mathrm{mg}$ of Pmab was administered intravenously by drip infusion. The patient then complained of dyspnea with shortness of breath and wheezing. Her oxygen saturation decreased to $88 \%$. Chemotherapy was immediately halted and oxygen, plus $250 \mathrm{mg}$ of aminophylline hydrate and $125 \mathrm{mg}$ of methylprednisolone sodium succinate, were given intravenously. As a result of this treatment, her symptoms gradually resolved within a few hours. Due to her successful recovery from IR, mFOLFOX6 without Pmab was administered the following week. No apparent symptoms were observed after this treatment. Diagnosing that the patient had suffered severe IR to Pmab, concomitant use of Cmab was attempted in the next course while monitoring vital signs. Three hundred and twenty $\mathrm{mg}$ of Cmab was slowly infused $(2.7 \mathrm{mg} /$ minute $)$ after premedication with $1.65 \mathrm{mg}$ of dexamethasone sodium phosphate, $20 \mathrm{mg}$ of famotidine and $50 \mathrm{mg}$ of Restamine calcium. No abnormal vital signs or IR symptoms were detected during administration. Other drugs were also administered without the appearance of any symptoms. Treatment was continued for seven courses due to no toxicities that might suggest the need for dose reduction or postponement, even though the patient experienced toxicities that included $\mathrm{G} 2$ dermatitis, G2 peripheral neuropathy, G2 dysgeusia, G2 thrombocytopenia, G1 anemia and G1 neutropenia. Marked tumor shrinkage (Fig. 2) allowed abdomino-perineal resection to be performed. Pathologically, the rectal wall was highly degenerated and showed fibrotic changes. However, live cancer
Fig. 2 Abdomino-pelvic CT scan after chemotherapy. A marked shrinkage of rectal tumor was demonstrated
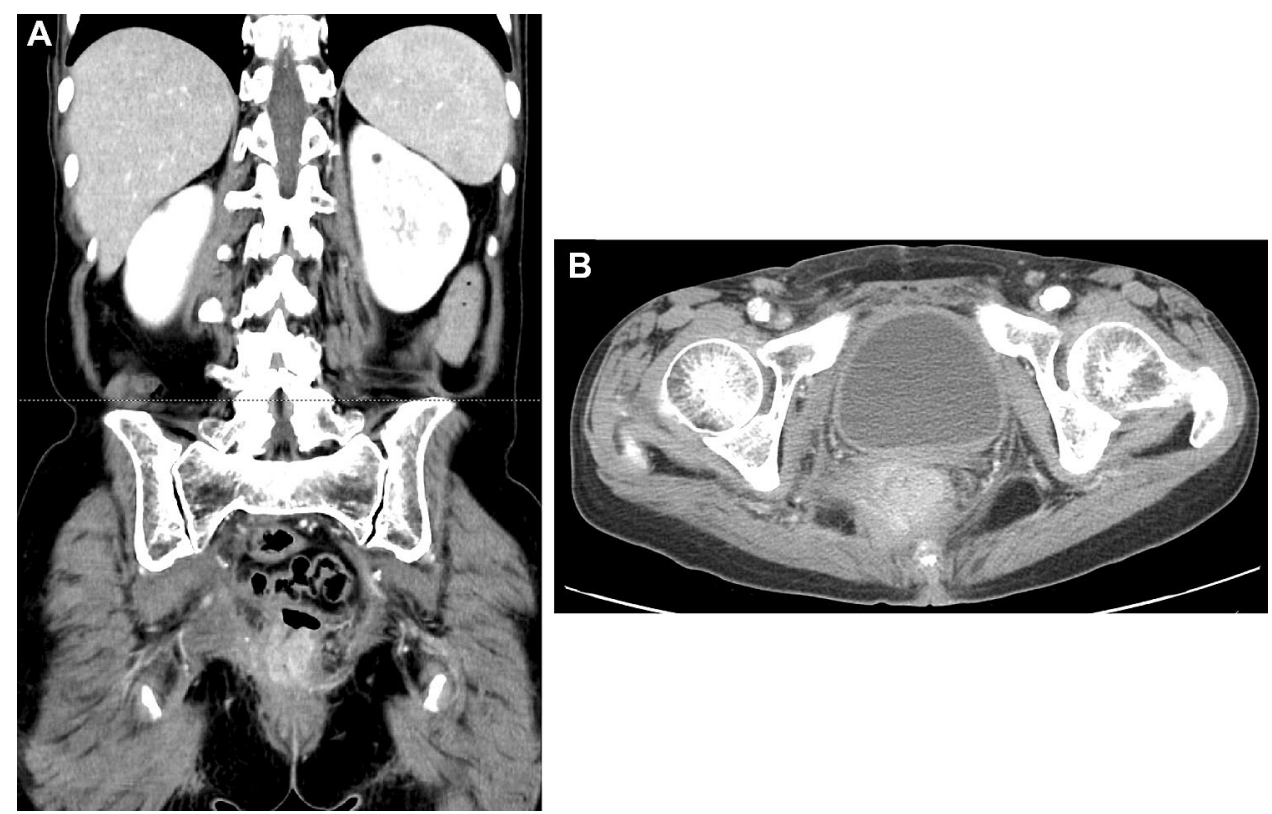
cells remained. Since these were found close to the surgical margin, she was diagnosed with pT4b (pelvic tissue), pN0, pStage II, pRM1, curB.

\section{Discussion}

Monoclonal antibody treatments that act on EGFR, including Cmab and Pmab, are recommended for Ras wild-typemetastatic colorectal cancer as candidates for first-line to third-line therapy [12-14]. Although cutaneous side effects are common, due to inhibition of EGFR expression in normal organs, certain prophylactic measures are commonly used, such as the use of moisturizing ointments or steroids, and oral intake of minocycline hydrochloride [15].

IR is a less common adverse event caused by antibodies to EGFR [2]. The fact that $90 \%$ of IRs occur during the first infusion despite antihistamic premedication suggests that these reactions occur without any IgE-mediated reaction [16]. It is possible that IRs to monoclonal antibodies are a reaction to human antichimeric antibodies or anti-human antibodies. In general, IR is more common with Cmab than Pmab. Possible rechallenge with $\mathrm{Cmab}$ may be due to differences in the reacting antibodies, even though no correlation between IR and these antibodies has been demonstrated. Another hypothesis for the mechanism that induces IR is that it is associated with the role of complement activation and the release of cytokines [17].

Our study has several limitations. First, mFOLFOX6 alone had a substantial therapeutic effect, and therefore, the benefit of the addition of Cmab were unclear. But previous study showed the superiority in the response rate of advanced colorectal cancer treated with FOLOX plus antiEGFR antibody compared to FOLFOX [18, 19]. Therefore, in this study, mFOLFOX plus Cmab potentially contributed to shrink tumor. Second, in patients with resectable colorectal liver metastasis, it is currently questionable whether resection of metastatic lesions after shrinking the tumor using Cmab plus mFOLFOX6 [20, 21]. But this is a report about locally advanced rectal cancer without any distant metastases. Resection of the shrinked primary lesion after chemotherapy containing anti-EGFR antibody might have improved her prognosis.

To our knowledge, this report is the second documentation of a case of successful rechallenge with Cmab after IR to Pmab. Since our case showed tumor shrinkage with concomitant use of Cmab and mFOLFOX6, she was able to undergo radical resection. Although IR is less frequent with Pmab, patients with severe IR to Pmab can be rechallenged with Cmab. However, further studies are needed to elucidate the pathogenesis and mechanisms of antibody-mediated IR and to gauge the safety of re-challenging with the same or different antibodies.

\section{Compliance With Ethical Standards}

Conflict of interest The authors declare that they have no conflicts of interest.

Research involving human participants and/or animals Formal consent is not required for this type of study. This article does not contain any studies with human participants or animals performed by any of the authors.

Informed consent Informed consent was obtained from the individual who participated in the study.

Open Access This article is licensed under a Creative Commons Attribution 4.0 International License, which permits use, sharing, adaptation, distribution and reproduction in any medium or format, as long as you give appropriate credit to the original author(s) and the source, provide a link to the Creative Commons licence, and indicate if changes were made. The images or other third party material in this article are included in the article's Creative Commons licence, unless indicated otherwise in a credit line to the material. If material is not included in the article's Creative Commons licence and your intended use is not permitted by statutory regulation or exceeds the permitted use, you will need to obtain permission directly from the copyright holder. To view a copy of this licence, visit http://creativecommons.org/licenses/by/4.0/.

\section{References}

1. Wong SF (2005) Cetuximab: an epidermal growth factor receptor monoclonal antibody for the treatment of colorectal cancer. Clin Ther 27:684-694

2. Cohenuram M, Saif MW (2007) Panitumumab the first fully human monoclonal antibody: from the bench to the clinic. Anticancer Drugs 18:7-15

3. Cunningham D, Humblet $Y$, Siena S et al (2004) Cetuximab monotherapy and cetuximab plus irinotecan in irinotecan-refractory metastatic colorectal cancer. N Engl J Med 351:337-345

4. Saltz LB, Meropol NJ, Loehrer PJ Sr et al (2004) Phase II trial of cetuximab in patients with refractory colorectal cancer that expresses the epidermal growth factor receptor. J Clin Oncol 22:1201-1208

5. Bonner JA, Harari PM, Giralt J et al (2006) Radiotherapy plus cetuximab for squamous-cell carcinoma of the head and neck. N Engl J Med 354:567-578

6. Gibson TB, Ranganathan A, Grothey A (2006) Randomized Phase III trial results of panitumumab, a fully human anti-epidermal growth factor receptor monoclonal antibody, in metastatic colorectal cancer. Clin Colorectal Cancer 6:29-31

7. Van Cutsem E, Peeters M, Siena S et al (2007) Open-label phase III trial of panitumumab plus best supportive care compared with best supportive care alone in patients with chemotherapy-refractory metastatic colorectal cancer. J Clin Oncol 25:1658-1664

8. Van Cutsem E, Siena S, Humblet Y et al (2008) An open-label, single-arm study assessing safety and efficacy of panitumumab in patients with metastatic colorectal cancer refractory to standard chemotherapy. Ann Oncol 19:92-98

9. Price TJ, Peeters M, Kim TW et al (2014) Panitumumab versus cetuximab in patients with chemotherapy-refractory wildtype KRAS exon 2 metastatic colorectal cancer (ASPECCT): 
a randomised, multicentre, open-label, non-inferiority phase 3 study. Lancet oncol 15:569-579

10. Saif MW, Peccerillo J, Potter V (2008) Successful rechallenge with panitumumab in patients who developed hypersensitivity reactions to cetuximab: report of three cases and review of literature. Cancer Chemother Pharmacol 63:1017-1022

11. Saif MW, Syrigos KI, Hotchkiss S et al (2009) Successful desensitization with cetuximab after an infusion reaction to panitumumab in patients with metastatic colorectal cancer. Cancer Chemother Pharmacol 65:107-112

12. Labianca R, Nordlinger B, ESMO Guidelines Working Group, et al. (2020) Primary colon cancer: ESMO Clinical Practice Guidelines for diagnosis, adjuvant treatment and follow-up. Ann Oncol 24 Suppl 6:vi64-72

13. Benson AB, Venook AP, Al-Hawary MM, et al. (2020) Colon Cancer. Version 2.2020. In: National comprehensive cancer network clinical practice guidelines in oncology (NCCN Guidlines). Available via DIALOG. https://www.nccn.org/professionals/physi cian_gls/pdf/colon.pdf. Accessed May 2020

14. Hashiguchi Y, Muro K, Saito Y et al (2020) Japanese Society for Cancer of the Colon and Rectum (JSCCR) guidelines 2019 for the treatment of colorectal cancer. Int J Clin Oncol 25:1-42

15. Kobayashi Y, Komatsu Y, Yuki S et al (2015) Randomized controlled trial on the skin toxicity of panitumumab in Japanese patients with metastatic colorectal cancer: HGCSG1001 study; J-STEPP. Future Oncol 11:617-627

16. Kang SP, Saif MW (2007) Infusion-related and hypersensitivity reactions of monoclonal antibodies used to treat colorectal cancer-identification, prevention, and management. J Support Oncol $5: 451-457$
17. van der Kolk LE, Grillo-López AJ, Baars JW et al (2001) Complement activation plays a key role in the side-effects of rituximab treatment. Br J Haematol 115:807-811

18. Bokemeyer C, Bondarenko I, Hartmann JT et al (2011) Efficacy according to biomarker status of cetuximab plus FOLFOX-4 as first-line treatment for metastatic colorectal cancer: the OPUS study. Ann Oncol 22:1535-1546

19. Douillard JY, Siena S, Cassidy L et al (2014) Final results from PRIME: randomized phase III study of panitumumab with FOLFOX4 for first-line treatment of metastatic colorectal cancer. Ann Oncol 25:1346-1355

20. Mise Y, Hasegawa K, Oba M, et al. (2019) EXPERT study: Randomized phase III trial of radical surgery and postoperative mFOLFOX6 versus perioperative mFOLFOX6 plus cetuximab in patients with KRAS wild-type resectable colorectal liver metastases (CLMs). J Clin Oncol 37(4_suppl):652

21. Bridgewater JA, Pugh SA, Maishman T et al (2020) Systemic chemotherapy with or without cetuximab in patients with resectable colorectal liver metastasis (New EPOC): long-term results of a multicentre, randomised, controlled, phase 3 trial. Lancet Oncol 21:398-411

Publisher's Note Springer Nature remains neutral with regard to jurisdictional claims in published maps and institutional affiliations. 